\title{
Effect of hypophosphatemia on the withdrawal of mechanical ventilation in patients with acute exacerbations of chronic obstructive pulmonary disease
}

\author{
YULIANG ZHAO, ZHIHAI LI, YINJUN SHI, GUNGKE CAO, FANYING MENG, WANG ZHU and GE YANG \\ Intensive Care Unit, The First People's Hospital of Xuzhou, Xuzhou, Jiangsu 221002, P.R. China
}

Received December 28, 2015; Accepted February 19, 2016

DOI: $10.3892 /$ br.2016.605

\begin{abstract}
Mechanical ventilation (MV) is a life-supporting modality frequently performed in critically ill patients to mechanically assist or replace spontaneous breathing. The aim of this study was to investigate the effect of hypophosphatemia on the withdrawal of MV in patients with acute exacerbations of chronic obstructive pulmonary disease (AECOPD). The medical records of 67 AECOPD cases, treated with MV between 2011 and 2013, were analyzed retrospectively. The patients were assigned to the hypophosphatemic and the normophosphatemic groups according to their serum phosphate levels (with the threshold of $0.87 \mathrm{mmol} / \mathrm{l}$ ). The results showed that the morbidity rate of hypophosphatemia in these AECOPD patients was $56.72 \%$. A significantly higher percentage of failure-to-wean from MV was observed in the hypophosphatemia group vs. the normophosphatemic group (34.21 vs. $10.34 \%, \mathrm{P}<0.05)$. Furthermore, hypophosphatemia was associated with respiratory muscular weakness, as reflected by a decrease in the tidal volume of spontaneous respiration, reduced static lung compliance and impaired pulmonary function, leading to weaning failure. The findings suggested that hypophosphatemia significantly affected the weaning from MV in AECOPD patients. Serum phosphorus levels may therefore serve as a reference index to determine the success of weaning.
\end{abstract}

\section{Introduction}

Mechanical ventilation (MV) is a life-supporting modality frequently performed in critically ill patients, such as those with acute exacerbations of chronic obstructive pulmonary disease (AECOPD), to mechanically assist or replace spontaneous breathing $(1,2)$. MV serves only to provide assistance for ventilation of the lungs, cannot reverse any underlying

Correspondence to: Dr Yuliang Zhao, Intensive Care Unit, The First People's Hospital of Xuzhou, 19 Zhongshan Road North, Xuzhou, Jiangsu 221002, P.R. China

E-mail: bt071h@163.com

Key words: hypophosphatemia, acute exacerbations of chronic obstructive pulmonary disease, mechanical ventilation, weaning disease process, but carries potential complications including acute lung/airway injury, acute respiratory distress syndrome, pneumothorax, and ventilator-associated pneumonia (3-5). Although there are no specific criteria may be generalized to all patients, withdrawal from MV, also known as weaning, should not be delayed unnecessarily $(6,7)$. Previous findings have indicated that several factors potentially affect the success of weaning from MV including phosphate imbalances or disorders (8-10). Nevertheless, clinical evidence regarding this issue is limited.

The aim of this retrospective study was to determine whether hypophosphatemia could serve as a predictor of success-to-wean from MV, with a focus on patients with AECOPD. We examined the medical records of 67 patients who received MV in the First People's Hospital of Xuzhou (Xuzhou, China), between 2011 and 2013. The patients were assigned to the hypophosphatemic and normophosphatemic groups according to their serum phosphate levels (with the threshold of $0.87 \mathrm{mmol} / \mathrm{l})$. Patient charts were reviewed and information was collected regarding medical anamnesis, physical examination, monitoring of records and biochemical analyses. The effect of hypophosphatemia on the withdrawal from MV in these AECOPD patients was evaluated.

\section{Materials and methods}

Patient information and allocation. Approval for this study was obtained from the Ethics Committee of the First People's Hospital of Xuzhou. A total of 67 patients with AECOPD, comprising of 41 men and 26 women, aged 52-81 years, hospitalized between 2011 and 2013, were included. The patients were mechanically ventilated and had either an endotracheal or tracheostomy tube. Of the 67 cases, 38 patients had serum phosphate levels $<0.87 \mathrm{mmol} / 1$ and were assigned to the hypophosphatemic group. The remaining 29 cases with a serum phosphate concentration $\geq 0.87 \mathrm{mmol} / 1$ constituted the normophosphatemic group. Differences in the forced expiratory volume in $1 \mathrm{sec}$ (FEV1) and in the vital capacity between the hypophosphatemic and normophosphatemic groups were $<70 \%$. Specifically, FEV1 accounted for $47.0 \%$, in the normophosphatemic group, and $48.1 \%$, in the hypophosphatemic group, of the predictive value $(\mathrm{F}=1.414$ under statistical processing, or less than tabular 3.12), with no significant difference between the two groups $(\mathrm{P}>0.05)$. In addition, the 
Table I. Comparison of weaning from mechanical ventilation in AECOPD patients from the hypophosphatemic and normophosphatemic groups.

\begin{tabular}{lcccc}
\hline Group & Total, no. & Failure-to-wean, no. & Success-to-wean, no. & Failure, $\%$ \\
\hline Hypophosphatemia & 38 & 13 & 25 & $34.21^{\mathrm{a}}$ \\
Normophosphatemia & 29 & 3 & 26 & 10.34 \\
Total & 67 & 16 & 51 & 23.88 \\
\hline
\end{tabular}

${ }^{\mathrm{a}} \mathrm{P}<0.05$ comparing to the normophosphatemic group. AECOPD, acute exacerbations of chronic obstructive pulmonary disease.

Table II. Comparison of respiratory parameters between the hypophosphatemic and normophosphatemic groups.

\begin{tabular}{lccc}
\hline Group & Tidal volume $(\mathrm{ml})$ & Minute ventilation volume $(\mathrm{ml})$ & Static lung compliance $\left(\mathrm{l} / \mathrm{cm} \mathrm{H}_{2} \mathrm{O}\right)$ \\
\hline Hypophosphatemia & $328^{\mathrm{a}}$ & 4,570 & $26^{\mathrm{a}}$ \\
Normophosphatemia & 368 & 4,850 & 39 \\
\hline
\end{tabular}

${ }^{\mathrm{a}} \mathrm{P}<0.01$ comparing to the normophosphatemic group.

Acute Physiology and Chronic Health Evaluation II scores in the two groups were comparable $(23 \pm 5.2$ and $21 \pm 4.9$, respectively; $\mathrm{P}>0.05$ ), confirming similar severity of illness between the two groups.

Data collection. Blood samples collected from all 67 cases were analyzed using a Hitachi 7600 automatic biochemical analyzer (Holliston, MA, USA). The obtained serum phosphate results were used in patient allocation, as stated above, for the normophosphatemic group $(\geq 0.87 \mathrm{mmol} / \mathrm{l})$ and the hypophosphatemic group $(<0.87 \mathrm{mmol} / \mathrm{l})$. Prior to weaning from ventilator support, synchronized intermittent mandatory ventilation was implemented, in which the ventilator breaths were synchronized with patient inspiratory effort. Patient respiratory parameters, including the tidal volume of autonomous respiration, the minute ventilation volume and the static lung compliance, were monitored and recorded.

Criteria for weaning success or failure. If a patient, $24 \mathrm{~h}$ after weaning at $\mathrm{PCO}_{2}<60 \mathrm{mmHg}$ and $\mathrm{PO}_{2}>60 \mathrm{mmHg}$, continued to maintain a respiratory rate of $<30$ times/min, normal cardiovascular function, along with no paradoxical respiration and no irritation, the weaning was considered successful; otherwise, as failure.

Statistical methods. Statistical analysis was performed with SPSS 10.0 software (SPSS, Inc., Chicago, IL, USA). Comparison of categorical data (\% failure-to-wean) between the hypophosphatemic and normophosphatemic groups was performed using the Pearson's Chi-square test. Measurable data were subjected to the F-test. $\mathrm{P}<0.05$ was considered to indicate a statistically significant difference.

\section{Results}

Weaning. Duration of MV applied to the 67 cases of AECOPD under examination varied from $21 \mathrm{~h}$ to 3.2 months, with an average of $75 \mathrm{~h}$. By the end of weaning from ventilator support, 7 cases succumbed.

Of the 67 AECOPD patients, 29 were cases of normal serum phosphate and 38 were cases of hypophosphatemia. Thus, the morbidity rate of hypophosphatemia was $56.72 \%$ in AECOPD patients treated with MV. In the normophosphatemic group, 26 cases were successfully weaned and 3 cases failed (10.34\%). By contrast, in the hypophosphatemic group, 25 cases were successfully weaned and 13 cases failed (34.21\%). The percentage of weaning failure was significantly higher in the hypophosphatemic vs. the normophosphatemic groups $(\mathrm{P}<0.05$; Table I).

Respiratory parameters. When synchronized intermittent mandatory ventilation was used, the tidal volume of spontaneous respiration was smaller in the normophosphatemic group than that in the hypophosphatemic group (368 and $328 \mathrm{ml}$, respectively, $\mathrm{P}<0.01)$. By contrast, the minute ventilation volume of the normophosphatemic group was similar to the hypophosphatemic group $(4,850$ and $4,570 \mathrm{ml}$, respectively, $\mathrm{P}>0.05)$. A significant difference was identified in static lung compliance between the two groups $\left(39 \mathrm{l} / \mathrm{cm} \mathrm{H}_{2} \mathrm{O}\right.$ and $26 \mathrm{l} / \mathrm{cm} \mathrm{H}_{2} \mathrm{O}$, respectively, $\left.\mathrm{P}<0.01\right)$. Results shown in Table II suggested that hypophosphatemia was associated with respiratory muscular weakness (a decrease in the volume of spontaneous respiration), reduced static lung compliance and impaired pulmonary function, leading to weaning failure in AECOPD patients.

\section{Discussion}

Hypophosphatemia is a common co-morbidity in critically ill patients including sepsis (11), often leading to increased mortality, prolonged duration of MV and intensive care unit (ICU) stay $(11,12)$. Some reports have suggested an association between respiratory illness and hypophosphatemia $(13,14)$, the latter of which significantly contributed to failure of 
weaning (15) and according to Haider et al (16), was an independent risk factor for mortality in critically ill patients. It was reported that the 24-h plasma phosphate level in some ICU patients after admission, especially those with respiratory failure, decreased to the reduced renal threshold of phosphate concentration (17).

While previous clinical studies showed that $20 \%$ of patients with chronic obstructive pulmonary disease had an occurrence of hypophosphatemia (1), our results demonstrate an even higher prevalence (56.7\%) in AECOPD patients under ventilator assistance. The percentage of weaning failure was significantly higher in the hypophosphatemic vs the normophosphatemic groups $(\mathrm{P}<0.05)$. Moreover, patients with hypophosphatemia were associated with respiratory muscular weakness, reflected by a decrease in the volume of spontaneous respiration, reduced static lung compliance and a loss of lung function, leading to weaning failure in AECOPD patients. These findings were consistent with those of previous reports $(2,9,10,18,19)$. The following pathogenetic mechanisms may be involved in the development of hypophosphatemia in AECOPD patients $(3,18,19)$ :

i) Insufficiency of phosphorus intake. Most AECOPD patients consumed less food when being treated with MV to assist breathing. Even for those who consumed an adequate amount of food, the phosphorus content of the foods may have been insufficient. Moreover, clinical therapy often focused on replenishing energy with vitamins $\mathrm{K}^{+}, \mathrm{Na}^{+}, \mathrm{Cl}^{-}$, as well as $\mathrm{Ca}^{++}$ and $\mathrm{Mg}^{++}$, while ignoring the supply of phosphorus.

ii) Imbalanced metastasis of serum phosphorus outside and inside cells. Patients with AECOPD often received intravenous injection of large amounts of glucose and insulin over a long period of time. They may also experience severe infection. These conditions may lead to higher cellular phosphorus absorption, negligible intracellular phosphorus-to-serum transfer, and acid-base imbalance. Under MV, respiratory alkalosis may produce a further decrease in serum phosphorus.

iii) Increase in serum phosphorus excretion. The use of diuretics, theophyllines and hormonic drugs may increase phosphorus excretion. Hypophosphatemia may lead to decreases in 2,3-diphosphoglyceric compound and ATP in erythrocytes (showing a shift to the left in an oxygen dissociation curve). It may critically reduced oxygen transfer from erythrocytes to tissues, resulting in tissue hypoxia, and the eventual failure of weaning. The results of our study show that hypophosphatemia causes respiratory muscular weakness (a decrease in the volume of autonomous respiration), reduction of static lung compliance and a loss of respiratory function, leading to weaning failure.

MV was a highly effective treatment in AECOPD patients suffering from severe respiratory failure. However, continuous application of a ventilator may result in uncontrolled infection, airway injury, and increased cost of treatment (2-5). Concurrently, there was a significant association between severe hypophosphatemia and the mortality of patients under ventilator support $(11,12)$. Additionally, hypophosphatemia affected the recovery of respiratory function, causing ventilator dependence, and, consequently, weaning failure. Clinics typically ignore this type of problem. Nevertheless, ventilator assistance providing support to underlying diseases is an independent factor in the deaths of patients infected with pseudomonas aeruginosa and having hematological tumor, low-phosphorus, low-protein and hyperlactatemia (20). Since the low-phosphorus factor prevails continuously in AECOPD patients using MV, the patients should be tested frequently and have phosphorus replenished according to test results, while constantly bearing in mind that excessive phosphorus intake is also harmful $(9,10)$.

In summary, our findings suggest that hypophosphatemia significantly affected the success of weaning from MV in patients with AECOPD. Serum phosphorus levels therefore serve as a reference index to determine the success of weaning.

\section{References}

1. No authors listed: International consensus conferences in intensive care medicine: Ventilator-associated Lung Injury in ARDS. This official conference report was cosponsored by the American Thoracic Society, The European Society of Intensive Care Medicine, and The Societé de Réanimation de Langue Française, and was approved by the ATS Board of Directors, July 1999. Am J Respir Crit Care Med 160: 2118-2124, 1999.

2. Hess DR: Approaches to conventional mechanical ventilation of the patient with acute respiratory distress syndrome. Respir Care 56: 1555-1572, 2011.

3. Yu YS: Theory and Practice of Modern Mechanical Ventilation. Beijing Union Medical University Press, Beijing, pp786-787, 2000.

4. Koulenti D, Blot S, Dulhunty JM, Papazian L, Martin-Loeches I, Dimopoulos G, Brun-Buisson C, Nauwynck M, Putensen C, Sole-Violan J, et al; EU-VAP/CAP Study Group: COPD patients with ventilator-associated pneumonia: Implications for management. Eur J Clin Microbiol Infect Dis 34: 2403-2411, 2015.

5. Chen L and Zhang Z: Clinical observation in CPOD patients with AECOPD. Shandong Med J 41: 25-26, 2001.

6. Matić I, Danić D, Majerić-Kogler V, Jurjević M, Mirković I and Mrzljak Vucinić N: Chronic obstructive pulmonary disease and weaning of difficult-to-wean patients from mechanical ventilation: Randomized prospective study. Croat Med J 48: 51-58, 2007.

7. Alía I and Esteban A: Weaning from mechanical ventilation. Crit Care 4: 72-80, 2000.

8. Alsumrain MH, Jawad SA, Imran NB, Riar S, DeBari VA and Adelman M: Association of hypophosphatemia with failure-to-wean from mechanical ventilation. Ann Clin Lab Sci 40: 144-148, 2010

9. Farah R, Khamisy-Farah R, Arraf Z, Jacobson L and Makhoul N: Hypophosphatemia as a prognostic value in acute exacerbation of COPD. Clin Respir J 7: 407-415, 2013.

10. Laaban JP, Grateau G, Psychoyos I, Laromiguière M, Vuong TK and Rochemaure J: Hypophosphatemia induced by mechanical ventilation in patients with chronic obstructive pulmonary disease. Crit Care Med 17: 1115-1120, 1989.

11. Shor R, Halabe A, Rishver S, Tilis Y, Matas Z, Fux A, Boaz M and Weinstein J: Severe hypophosphatemia in sepsis as a mortality predictor. Ann Clin Lab Sci 36: 67-72, 2006.

12. Sakhawey A, Alawady S and Razek AA: Effect of phosphate level on the outcome of critically ill patients in the intensive care unit. J Am Sci 11: 82-88, 2015.

13. Fisher J, Magid N, Kallman C, Fanucchi M, Klein L, McCarthy D, Roberts I and Schulman G: Respiratory illness and hypophosphatemia. Chest 83: 504-508, 1983.

14. Rohini K, Bhat S, Srikumar PS and Mahesh Kumar A: Assessment of serum calcium and phosphorus in pulmonary tuberculosis patients before, during and after chemotherapy. Indian J Clin Biochem 29: 377-381, 2014.

15. Agusti AG, Torres A, Estopa R and Agustividal A: Hypophosphatemia as a cause of failed weaning: The importance of metabolic factors. Crit Care Med 12: 142-143, 1984.

16. Haider DG, Lindner G, Wolzt M, Ahmad SS, Sauter T, Leichtle AB, Fiedler GM, Fuhrmann V and Exadaktylos AK: Hyperphosphatemia Is an Independent Risk Factor for Mortality in Critically Ill Patients: Results from a Cross-Sectional Study. PLoS One 10: e0133426, 2015. 
17. Moran JL, Solomon PJ, Yeung KWA, Pannall PR, John G and Eliseo A: Phosphate metabolism in intensive care patients with acute respiratory failure. Crit Care Resusc 4: 93-103, 2002.

18. Fiaccadori E, Coffrini E, Fracchia C, Rampulla C, Montagna T and Borghetti A: Hypophosphatemia and phosphorus depletion in respiratory and peripheral muscles of patients with respiratory failure due to COPD. Chest 105: 1392-1398, 1994.

19. Fiaccadori E, Coffrini E, Ronda N, Vezzani A, Cacciani G, Fracchia C, Rampulla C and Borghetti A: Hypophosphatemia in course of chronic obstructive pulmonary disease. Prevalence, mechanisms, and relationships with skeletal muscle phosphorus content. Chest 97: 857-868, 1990.
20. Jeddi R, Ghédira H, Ben Amor R, Turki A, Kacem K, Ben Abdennebi Y, Ben Lakhal R, Aissaoui L, Ben Abid H, Bel Hadjali Z and Meddeb B: Risk factors of septic shock in patients with hematoligic malignancies and Pseudomonas infections. Hematology 16: 160-165, 2011. 